\title{
EVALUATION OF INTEL i7 CORE PROCESSOR
}

\author{
Prerna Setia*1, Nishchal $^{2}$, satvinder ${ }^{3}$ \\ *1,2,3Electronics and Communication Engineering, Dronacharya College of Engineering, Gurgaon. \\ *1prernasetia977@gmail.com, ${ }^{2}$ nickbloom3@gmail.com, ${ }^{3}$ singh.satvinder90@gmail.com
}

\section{*Corresponding Author: -}

Email: prernasetia977@gmail.com

\begin{abstract}
: -
The Intel Nehalem microarchitecture that encompasses the Core i7 class of processors uses a $45 \mathrm{~nm}$ fabrication process for different processors in the Core i7 family. Besides using the power consumption benefits of $45 \mathrm{~nm}$, Intel made some dramatic changes in the Nehalem microarchitecture to offer new features and capabilities in the Core i7 family of processors.The paper presented over here is a complete exposure to the precisely used technologies in Intel i7 core processor.Intel i7 processor is a newly available and latest core processor present in the market. It uses all the new technologies present with in the corporative world. It is redesigned by a high repetitive quality of software technocrats so that it is sophisticated form of all of the core processors present.Intel Core i7 usually applies to all families of desktop and laptop 64-bit x86-64processors which uses the Westmere, Nehalaem, Ivy, Sandy Bridge and the Haswell microarchitectures. The Core i7 brand mostly targets all the business and high-end consumer markets for both desktop and laptop computers, and is distinguished from the (entry-level consumer) Core i3, (mainstream consumer) Core i5, and (server and workstation) Xeon brands.

In the first three microarchitecture generations of Intelbrand, Core i7 has family members using two distinct system-level architectures, and therefore two distinct. In each of the generation, the highest-performing Core i7 processors uses same socket and QPI-based architecture as the low-end Xeon processors of the previous generation, while the lowerperforming Core i7 processors uses the same socket and the PCIE/DMI/FDI architecture as was in the Core i5.

Intel firstly introduced the Core i7 in late 2001 with the name Nehalem-based BloomfieldQuad-core processor. In 2009 , the new Core 7 models based on the Lynnfield the Nehalem-based, desktop quad-core processor and the Clarksfield the Nehalem-based quad-core mobile was added, andIn January 2010 the models based on the Arrandale dual-core mobile processor were also Nehalem-based added too. The first six-core processor in the Core lineup is the Nehalem-based Gulftown, which was launched on March 16, 2010. Both the regular Core 77 and the Extreme Edition are advertised as five stars in the Intel Processor Rating
\end{abstract}

\section{(c) $(\$)$}




\section{INTRODUCTION: -}

"Intel" all are well aware of it is the multinational company that comes with new techonolgical developed software, processors that are in great demand in the market because of their higher and efficient compactibility, advanced response towards the users demand. Now, the question arise why only Intel core? .. Intel Penrynmicroarchitecture includes Core 2 family of the processors which was the first mainstream. Intel microarchitecture isbased on the $45 \mathrm{~nm}$ fabrication process. This allows Intel to make a higher performance range of processorsthat rapidly consumes similar or less power than the previously generation processors. Intel Core basically is a brand name which Intel uses for the various range of mid to high-end consumers and business-based microprocessors. Generally, processors sold in the name of Core are more powerful variants in the market of the same processors as marketed entry-level Celeron and Pentium. On the other hand, identical or more advanced versions of Core processors are moreover sold as Xeon processors for server and workstation market. As of 2013, the current Core processors includes the latest launched Intel Core i7, Intel Core i5, and Intel Core i3, and older Intel Core 2 Solo, Intel Core 2 Duo, Intel Core 2 Quad, and Intel Core 2 Extreme lines.Intel i7 core processor is one of the leading processor in the world it uses all of its advanced technology present. It is bit costlier than other processors present over but implies with the best and efficient work done

Nehalem the Intel microarchitecture that inhibits the Core i7 class of processors which uses a $45 \mathrm{~nm}$ fabrication process for the different processors in Core i7 family. In addition to, using the power consumption benefits of $45 \mathrm{~nm}$, Intel has made some of the dramatic changes in the Nehalem microarchitecture (Intel) to offer all the new features and capabilities presided in the Core i7 family.

\section{TECHNICAL ASPECTS: -}

The new features indulged in i7 core processors are as above: -

(a) Developed Microarchitecture:-
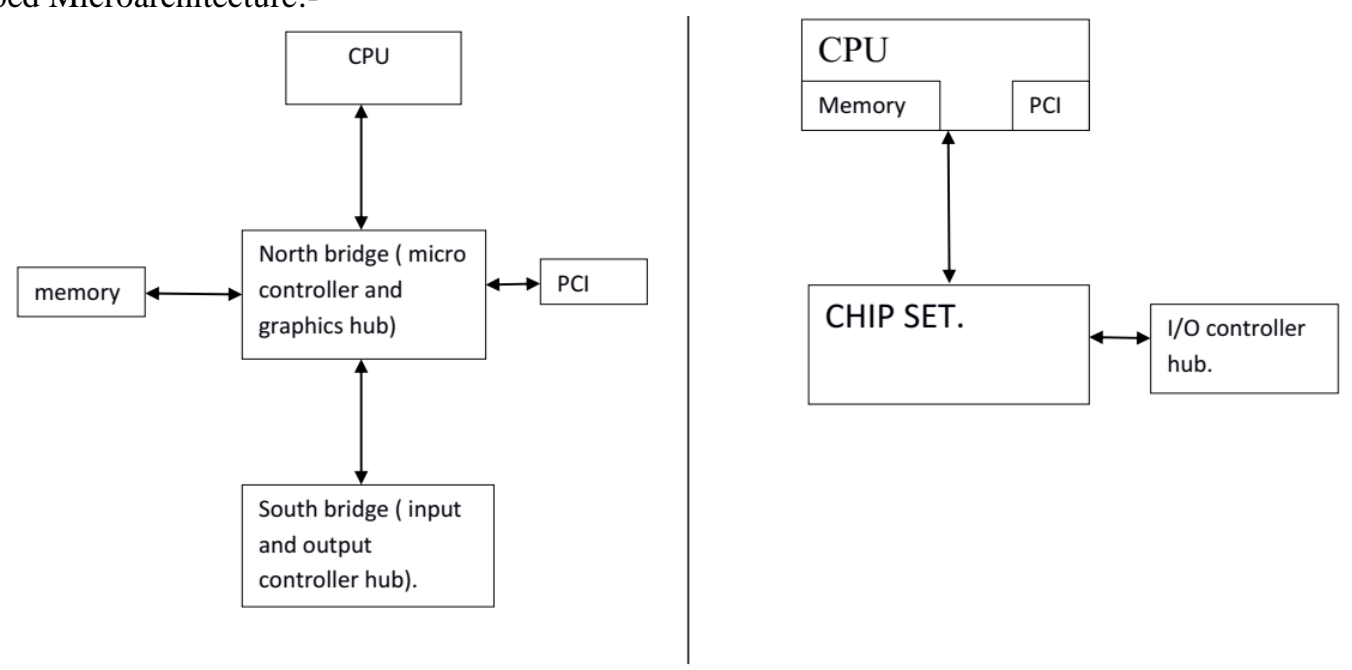

PREVIOUS MICRO ARCHITECTURE

NEHLAEM/ I7 MICRO ARCHITECTURE

In the previous intel microarchitectures as is shown above there are basically three main parts of the microarchitecture (a) CPU (Central processiong unit) (b) North bridge also called as micro controller and graphics hub. (c) South bridge also called as input and output controller hub. Both the memory and pci are connected to the north bridge whereas the cpu was freed increasing the complicity and however the i/o controller was connected to the south bridge. But in the nehaleam or i7 core processor the both memory and pci are connected to the CPU and the chipset is made in order to handle over the all i/ostreams. Thus, the complexity is reduced in a much effective way and the same way a lot of memory space is also increased a lot.

(b) Quick path interconnect is done with higher processor micro controller: -
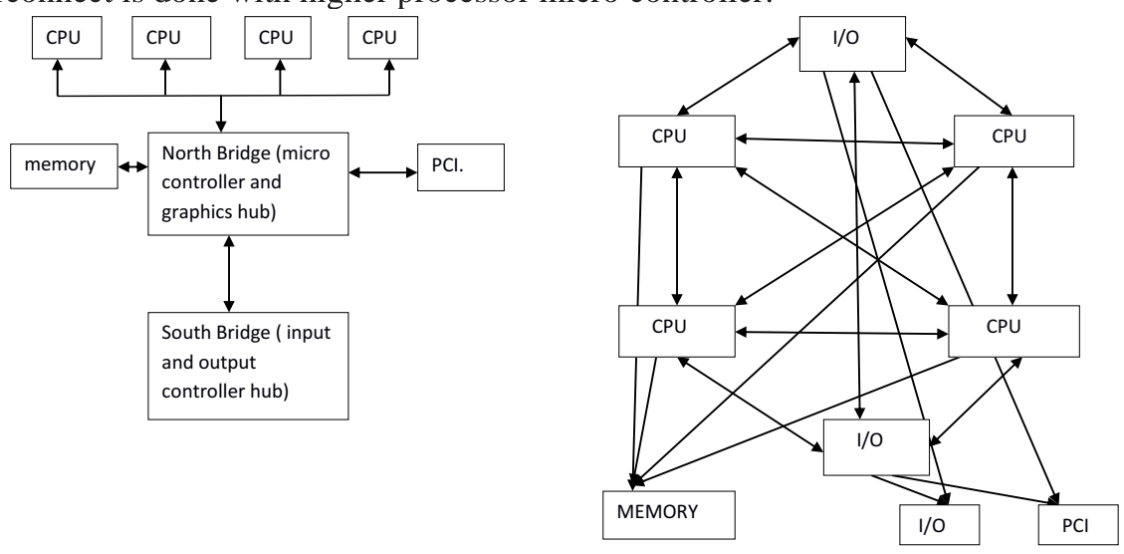
The Nehalem microarchitecture (core i7) integrated the memory controller and also introduced the high- speedQPI databus. In Nehalem-based multiprocessor system, each of the CPU has to access the local memory but they can also access the memory that is local one to other CPUs by the help of QPI transactions. For example, one of theCore i7 processor can access memory region from the local to another processor through one direct hop or through multiple hops.

(c) Intel turbo boost technology boosts CPU performance: -A performance boost is to provide for the lightly threaded applications and also optimizes processor power consumption. Intel knows introduced a new feature called Intel Turbo Boost. Intel Turbo Boost is a recently launched an innovative feature that automatically allows active processor cores to run faster than the base operating frequency when certain conditions are met.

(d) high quality of catch latency: - Cache is a block which has high-speed memory for the temporary data storage that is located on the same silicon chip as the CPU. If for a instance the single processing core in a multicore CPU, requires the specific data while it executes an instruction set, it firstly searches for the data in the local caches (L1 and L2). If the data is not available in the cache, its known as a cache- miss, after it accesses the larger L3 cache.

\section{Conclusion: -}

The advantages surely will make avery little difference in overall performance. It is correctly said that the intel core i7 contains all the main and scientific features one can contain and on other hand it is bit costlier than other processors because of the embedded technologies which has a great impact on the consumers. But it has a disadvantage to that it has a less clocking speed which makes it difficult to understand and utilize too.

When I reviewed the six-core Core i7 4960X a little surprised thing came in to notice that clock-for- clock Ivy Bridge -E is slower than the Sandy-Bridge-E generation. Advantages of intel core i7 processor: -

1. You get Quad Channel memory compatibility

2. Driving multiple graphics cards could favor Core i $7820 \mathrm{~K}$ as the X79 chipset has 40 PCI Express Gen 3.0 lanes.

\section{References: -}

[1]."DesktopProcessors"(http://www.intel.com/products/desktop/processors/index.htm?iid=processors_bod y+dt_core). Intel.com. Retrieved 2010-12-13.

[2]."Intel Launches Devil's Canyon and Overclockable Pentium: i7-4790K, i5-4690K and G3258" (http://www.anandtech.com/show/8079/intel-launchesdevils- canyon-and-overclockable-pentium-i74790k-i54690kand-g3258). Anandtech. 3 June 2014. Retrieved 29June 2014.

[3]."Intel already phasing out first quad-core CPU" (http://www.tgdaily.com/content/view/33752/135/). TG Daily. Retrieved 2007-09-07.

[4]."Intel to discontinue older Centrino CPUs in Q1 08" (http://www.tgdaily.com/content/view/34115/122/). TG Daily. Retrieved 2007-10-01.

[5]."Support for the Intel Core Duo Processor" (http://www.intel.com/support/processors/mobile/coreduo/). Intel.com. Retrieved 2010-12-13.

[6]."Support for the Intel Core Solo processor" (http://www.intel.com/support/processors/mobile/coresolo/). Intel.com. Retrieved 2010-12-13.

[7]."Intel Microarchitecture" (http://www.intel.com/technology/architecture-silicon/core/). Intel.com. Retrieved 2010-1213.

[8]."Intel Core2 Solo Mobile Processor - Overview" (http://www.intel.com/products/processor/core2solo/). Intel.com. Retrieved 2010-12-13.

[9]."Intel Core2 Duo Processor: Upgrade Today" (http://www.intel.com/products/processor/core2duo/). Intel.com. Retrieved 2010-12-13.

[10]. "Intel Core2 Duo Mobile Processor" (http://www.intel.com/products/processor/core2duo/mobile/). Intel.com. Retrieved 2010-12-13. 